\title{
Effects of Cold Atmospheric Pressure Plasma on Accelerating Acute Wound Healing: A Comparative Study among 4 Different Treatment Groups
}

\author{
Akio Nishijima1,2,3*, Takahiro Fujimoto ${ }^{1,3}$, Takamichi Hirata1, Junko Nishijima ${ }^{1,3}$ \\ ${ }^{1}$ Department of Medical Engineering, Faculty of Engineering, Tokyo City University, Tokyo, Japan \\ ${ }^{2}$ Department of Plastic and Reconstructive Surgery, University of Tsukuba, Ibaraki, Japan \\ ${ }^{3}$ Clinic F, Tokyo, Japan \\ Email: *handbal1016@yahoo.co.jp
}

How to cite this paper: Nishijima, A., Fujimoto, T., Hirata, T. and Nishijima, J. (2019) Effects of Cold Atmospheric Pressure Plasma on Accelerating Acute Wound Healing: A Comparative Study among 4 Different Treatment Groups. Modern Plastic Surgery, 9, 18-31.

https://doi.org/10.4236/mps.2019.91004

Received: January 4, 2019

Accepted: January 21, 2019

Published: January 24, 2019

Copyright $\odot 2019$ by author(s) and Scientific Research Publishing Inc. This work is licensed under the Creative Commons Attribution International License (CC BY 4.0).

http://creativecommons.org/licenses/by/4.0/

\begin{abstract}
Cold atmospheric pressure plasma (CAP) has been expected to be effective for wound healing among plasma medicines and has been attracting attention. The aim of this retrospective clinical study was to evaluate and compare the effects of CAP on acute wounds created by a fractional $\mathrm{CO}_{2}\left(\mathrm{FXCO}_{2}\right)$ laser on four treatment groups. Methods: This study was conducted between September 2017 and November 2018. Radiated surface wounds were created using a $\mathrm{FXCO}_{2}$ laser on four identically-sized regions on the medial side of the left forearm of 12 healthy volunteer subjects, for a total of 48 treatment regions. Each region was then randomly assigned to one of four groups: 1) no treatment (control group), 2) irradiated CAP for 60 seconds (plasma group), 3) topical application of ointment containing betamethasone valerate (steroid group), or 4) two to three sprays of basic fibroblast growth factor (bFGF group). The primary outcome of the skin was the change in absolute values of lightness index $\left(L^{*}\right)$, redness index $\left(a^{*} I\right)$ of $L^{*} a^{*} b$ color space, arithmetical mean roughness $(\mathrm{Ra})$, and mean melanin concentration. The secondary outcome was the clinical condition of the wounds, including inflammation, crust formation, and hyper/hypo-pigmentation on the recovering skin surface relative to the surrounding intact skin. Each item was evaluated at post-treatment $0,1,3,7,14,28$ days and 1 year. Results: Although no significant difference was observed among all groups, the CAP group exhibited more rapid recovery regarding $\mathrm{a}^{\star} \mathrm{I}$ and Ra. No side-effects were observed in the CAP group. Conclusion: CAP is considered to accelerate healing of irradiated wounds via anti-inflammatory activity. Our study confirmed the effectiveness and safety
\end{abstract}


of CAP which is devoid of side-effects of conventional therapies, and may aid in future development of medical plasma therapies.

\section{Keywords}

Cold Atmospheric Pressure Plasma, Plasma Medicine, New Energy Device, Wound Healing, Skin Activation

\section{Introduction}

In recent years, plasma treatment for wound healing has been attracting attention, with a rapid increase in the interest in medical devices based on cold atmospheric pressure plasma (CAP) [1] [2]. Since Laroussi first reported the use of atmospheric pressure plasma in 1996 [3], CAP has been experimentally investigated for several biomedical applications. However, some of the most potentially important therapeutic roles played by reactive oxygen and nitrogen species in medical interventions have emerged only in the past decade [4]. In clinical practice, the antibacterial activity of medical plasma has been noted, especially for the treatment of chronic ulcers such as venous stasis skin ulcers or diabetic foot gangrene [5]. However, to our knowledge, there has been no report on the effectiveness of CAP for acute human wounds using a three-dimensional skin analyzer in a randomized controlled trial.

Fractional $\mathrm{CO}_{2}\left(\mathrm{FXCO}_{2}\right)$ laser therapy has been used for skin resurfacing. This treatment has been successful in improving texture, acne scaring, color and wrinkles [6] [7] [8]. However, the downtime after irradiation, such as the period with redness and/or pigmentation of the irradiated skin, is of concern. For post-irradiation treatment, topical application of ointments, including steroids or petrolatum [9], basic fibroblast growth factor (bFGF) sprays, or gels containing fullerene [10] have been applied, as well as protection from solar radiation. However, a standard treatment has yet to be established, and the method chosen depends on the treating surgeon because each has merits and demerits.

The aim of this clinical study was to evaluate the effects of CAP on acute wounds created by a $\mathrm{FXCO}_{2}$ laser. Herein, we report the first randomized controlled trial comparing four different treatment groups to evaluate the effects of CAP on acute human wounds with objective methods for treatment outcome evaluation.

\section{Materials and Methods}

\subsection{Study Design}

This retrospective study was conducted between September 2017 and November 2018 to compare treatment outcomes of four identically-sized irradiated regions on the left forearm of healthy volunteer subjects. The protocol was conducted adhering ethical principles of the Declaration of Helsinki and approved by the 
institutional review board of Tokyo City University (IRB number: 413), and all subjects provided written informed consent.

\subsection{Subjects}

Twelve healthy volunteer subjects ( 9 women and 3 men) with a mean age of 34.3 \pm 12.1 years from the Tokyo City University and Clinic F in metropolitan Tokyo were enrolled. The subjects were required to adhere to the following requirements throughout the study: they were not to use local, systemic, or other medications, such as moisturizers or antibiotics; and they were to wear long sleeves to protect their skin from solar radiation. Subjects were excluded if they were unwilling to be assigned to any of the approaches.

\subsection{Randomization}

Each of the 12 subjects received irradiation on four areas of the same size $(1.5 \times$ $2.0 \mathrm{~cm}$ ) on the medial side of the left forearm, making a total of 48 lesions. Each lesion was created using a fractional $\mathrm{CO}_{2}\left(\mathrm{FXCO}_{2}\right)$ laser therapy device (SmartXide DOT RT ${ }^{\mathrm{R}}$, DEKA M.E.L.A.srl, Calenzano, Italy) with a single shot at an output power of $10 \mathrm{~W}$, pulse width of $600 \mu \mathrm{s}$, dot spacing of $650 \mu \mathrm{m}$, and stack of 2. The sample size was defined as $4 \times 4$, based on the Latin square method, because we conducted a randomized study for four regions for each subject. The minimum necessary sample number was determined to be 48 sites in 12 subjects, considering collectible number of data in the institutions.

\subsection{Treatment Protocol}

Immediately after $\mathrm{FXCO}_{2}$ laser irradiation, each of the four regions on the subjects' arms was subjected to one of four different treatments: CAP jets (the CAP group), steroid and antibiotic ointment (steroid group), basic fibroblast growth factor (bFGF group), or no treatment (control group). These interventions were applied only once, and were randomly allocated to each region using SAS software v9.4 (SAS Institute, Cary, NC, USA).

The CAP group was subjected to a CAP jet (described below) immediately after $\mathrm{FXCO}_{2}$ irradiation (Figure 1(a) and Figure 1(b)). Plasma treatment was performed by moving the visible tip of the plasma jet at $10 \mathrm{~mm}$ from the skin repeatedly over the entire target area for 60 seconds, in accordance with the previously reported laser radiation method for clinical practice [11] [12]. The steroid group received topical application of ointment containing betamethasone valerate and gentamicin sulfate (Rinderon-VG ${ }^{\oplus}$, Shionogi \& Co., Ltd, Tokyo, Japan). The bFGF group received 5 sprays of aerosolized trafermin (genetical recombination) (Fiblast ${ }^{\oplus}$ Spray, Kaken Pharmaceutical Co., Ltd, Tokyo, Japan) at approximately $5 \mathrm{~cm}$ apart from the wound area. Total trafermin was $1.0 \mu \mathrm{g} / \mathrm{cm}^{2}$ in the wound. All interventions were done only once after $\mathrm{FXCO}_{2}$ irradiation. Sincethe subjects were wearing an eye mask, they were not aware of which treatment was applied to which site. 

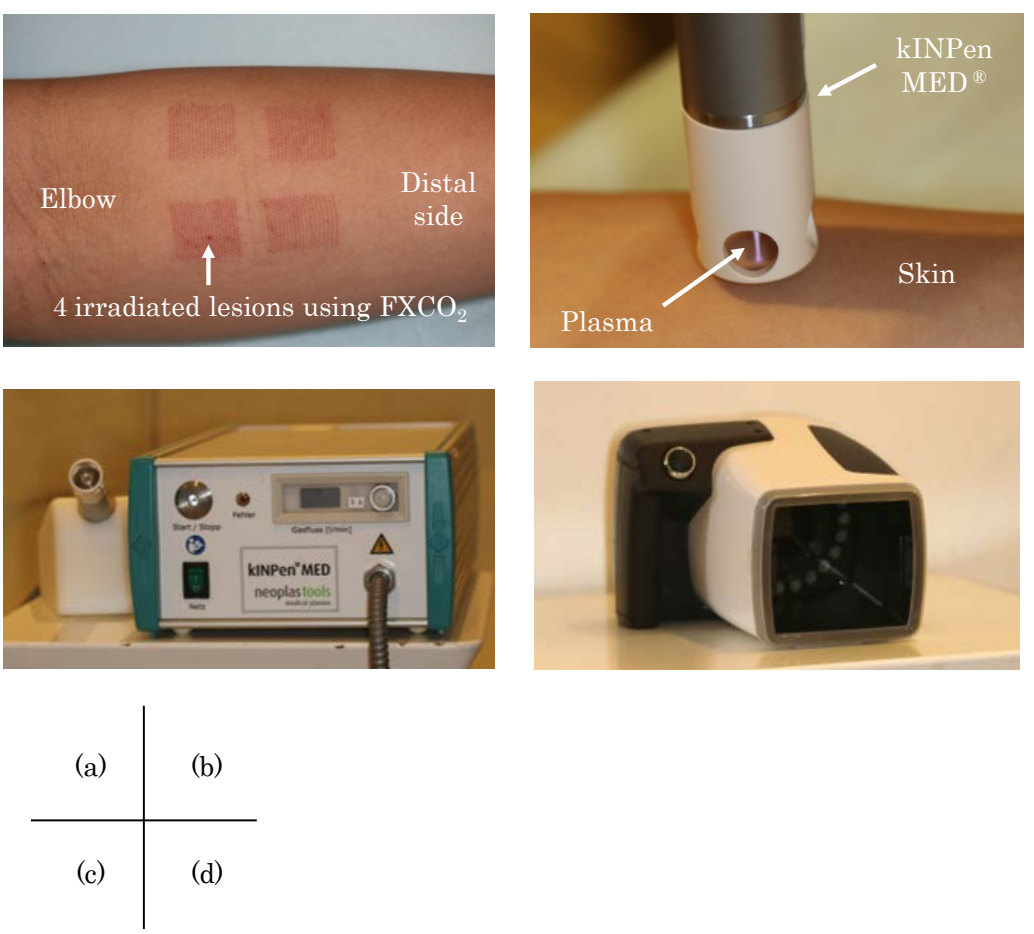

Figure 1. Wounds created by laser irradiation and equipment used in our study. (a) Photograph of the medial side of the left lower forearm immediately after irradiation by fractional $\mathrm{CO}_{2}$ laser; (b) Cold atmospheric pressure plasma (kINPen $\mathrm{MED}^{\circledR}$ ) jet radiation; (c) The entire kINPen MED ${ }^{\circledR}$ device; (d) Three-dimensional skin analyzer (ANTERA 3 $\mathrm{D}^{\mathrm{TM}}$ ).

\subsection{Cold Atmospheric Pressure Plasma Treatment}

Plasma was applied to the target region with CAP jets using a kINPen MED ${ }^{\circ}$ (INP Greifswald/neoplas tools GmbH, Greifswald, Germany) (Figure 1(c)). The device used in this study fulfilled the technical requirements for medical use, and consisted of a hand-held unit for the generation of gas discharge under atmospheric pressure conditions and a direct current power supply unit. A pin-type electrode ( $1 \mathrm{~mm}$ in diameter) was mounted at the center of a ceramic capillary (inner diameter: $1.6 \mathrm{~mm}$ ). A high voltage of $2-3 \mathrm{kV}_{\mathrm{pp}}$ at a frequency of $1 \mathrm{MHz}$ was periodically applied to the pin electrode $\left(f_{\text {rep }}=2.5 \mathrm{kHz}\right.$, plasma duty cycle $=$ 1:1). Plasma was generated at the tip of the central electrode and expanded out into the air surrounding the nozzle. The system was operated with argon gas at flow rates between 4 and $6 \mathrm{~L} / \mathrm{m}$. Under these working conditions, the plasma jet outside the ceramic capillary was approximately $10 \mathrm{~mm}$ long [1].

\subsection{Photographic Analysis}

Before and after the treatment, a 3D skin analyzer (ANTERA 3D ${ }^{\mathrm{TM}}$, Miravex Co., Ltd, Ireland) was used to record changes in the irradiated areas and to assess the process of wound healing (Figure 1(d)). The camera on the analyzer was equipped with $24 \mathrm{LED}$ lights of different wavelengths. During imaging, the camera detected the reflected light from different directions, which was then 
processed by a personal computer connected to the camera to obtain color tone data and morphological data displayed as 2- and 3D images. This allowed for quick quantitative measurements [13] [14] [15].

\subsection{Outcome Measurements}

To evaluate the process of wound healing, lightness, redness (flare), roughness, and pigmentation on the skin surface were measured by the 3D skin analyzer (ANTERA $3 \mathrm{D}^{\mathrm{TM}}$ ). The lightness ${ }^{\star}$ index $\left(\mathrm{L}^{\star} \mathrm{I}\right)$ of $\mathrm{L}^{\star} \mathrm{a}^{\star} \mathrm{b}^{\star}$ color space was used to evaluate lightness, ranging in values from 0 to 100 . The $a^{*}$ index $\left(a^{\star} I\right)$ of $L^{*} a^{*} b^{*}$ color space was used to evaluate redness, with higher values of $\mathrm{a}^{\star} \mathrm{I}$ indicated by more red/violet components and smaller values indicated by more blue/green components. Roughness of the skin was evaluated by the arithmetical mean roughness $(\mathrm{Ra})$, presented as the average of the values within the sampling region. $\mathrm{Ra}$ is a relatively stable parameter, which is not affected by a single unique value. Pigmentation was evaluated as the mean melanin concentration, as displayed on the monitor of ANTERA 3 ${ }^{\mathrm{TM}}$.

The evaluation items were measured immediately before and after $\mathrm{FXCO}_{2}$ laser irradiation, and at Days 1, 3, 7, 14, 28, and at 1 year after treatments. The primary outcome was the change in values of $L^{\star} I, a^{\star} I, R a$, and mean melanin concentration. The secondary outcome was the clinical condition of the wounds, relative to the intact skin, focusing on inflammation, crusting, hyper-pigmentation, hypo-pigmentation, and aesthetics. Healing results were comprehensively evaluated based on clinical findings such as color and dermal structure of recovering skin surface relative to the surrounding untreated areas. Aesthetic evaluation was based on aesthetic satisfaction assessment using the aesthetic numeric analogue scale (ANA-scale) [16] ranging between " 0 " and " 10 ", where " 0 " indicates the worst aesthetic outcome possible and " 10 " indicates an ideal aesthetic result. In our study, only " 10 " was defined as a "good result" to be reported [11]. Primary and secondary outcomes were evaluated in a blinded manner by a different clinician from the one who performed the treatments.

\subsection{Statistical Analysis}

No data from any of the 12 subjects were excluded from any analyses. Categorical data were summarized as the frequency, and continuous data as the mean and standard deviation (SD). Comparisons between groups were performed using the Mann-Whitney $U$ test or one-way analysis of variance (ANOVA) for continuous variables. All statistical analyses were performed with SAS 9.4 (SAS Institute, Cary, NC, USA). P-value of less than 0.05 was considered significant.

\section{Results}

\subsection{Primary Outcomes}

The relative skin lightness index of the steroid group demonstrated the earliest improvement, and the other groups had almost equivalent improvement speed 
(Figure 2). At the end point of Day 28, all groups had skin lightness values higher than those before treatment. The highest skin lightness was the steroid group at Day $28\left(L^{\star} I=71.7\right)$.

The $\mathrm{a}^{\star}$ Index $\left(\mathrm{a}^{\star} \mathrm{I}\right)$ of the control group at Day 1 indicated greater redness $\left(\mathrm{a}^{\star} \mathrm{I}\right.$ $=17.0)$ compared with that immediately after $\mathrm{FXCO}_{2}$ irradiation $\left(\mathrm{a}^{\star} \mathrm{I}=16.5\right)$, that of the bFGF group remained the same $\left(\mathrm{a}^{\star} \mathrm{I}=16.5\right)$, and the plasma $\left(\mathrm{a}^{\star} \mathrm{I}=\right.$ 15.2) and steroid $\left(\mathrm{a}^{*} \mathrm{I}=14.8\right)$ groups exhibited reduced redness (Figure 3$)$. From Day 3, all groups showed similar time-course improvement, all groups had almost the same value at Day 14 .

The arithmetical mean roughness $(\mathrm{Ra})$, representing increased post-radiation swelling immediately after $\mathrm{FXCO}_{2}$ laser radiation, improved at Day 1 (Figure 4). The Ra increased until Day 7, along with crust formation, and then decreased to a similar level as that before irradiation at Day 28. The earliest improvement in Ra was in the steroid group, the next was the plasma group.

The mean melanin concentration was 0.53 at Day 1 and the earliest reduction in the steroid group. The other 3 groups had similar time course changes (Figure 5). No groups had severe hyper-pigmentation.

Figure 6 shows the time-course changes of a representative case by the $3 \mathrm{D}$ skin analyzer. Values of $L^{\star} I$ and $a^{\star} I$ were calculated using the color images from the $3 \mathrm{D}$ skin analyzer. For all evaluation items, no significant differences were found among the 4 groups at each post-treatment time points.

\subsection{Secondary Outcomes (Table 1)}

Inflammation in the CAP group and steroid group was slightly less than the other two groups by Day 14, although all groups showed similar improvement at Day 28. Crusting was similar among the 4 groups. Hyper-pigmentation was slightly less in the steroid group, while no significant differences were observed among the other groups. Hypo-pigmentation was not observed in any group. No precancerous change was noted on the treated sites in any group at 1 year after CAP treatment. There were no notable differences at Day 28 and 1 year with all groups achieving good results.

\section{Discussion}

\subsection{Plasma Medicine}

Medical plasma has two major effects: antibacterial activity and tissue activation. In clinical practice, the antibacterial activity of medical plasma has been attracting attention, especially for the treatment of chronic ulcers such as venous stasis skin ulcers or diabetic foot gangrene [5]. Bacterial strains associated with chronic ulcers include Pseudomonas, Klebsiella, Staphylococci, and Micrococci, which are often difficult to treat in clinical settings. Many types of bacteria and fungi that are categorized as physiological or pathological skin flora are highly susceptible to plasma treatment [17]. In this study, we focused on biological tissue activation effects of medical plasma in acute phase wounds. The improvement in 


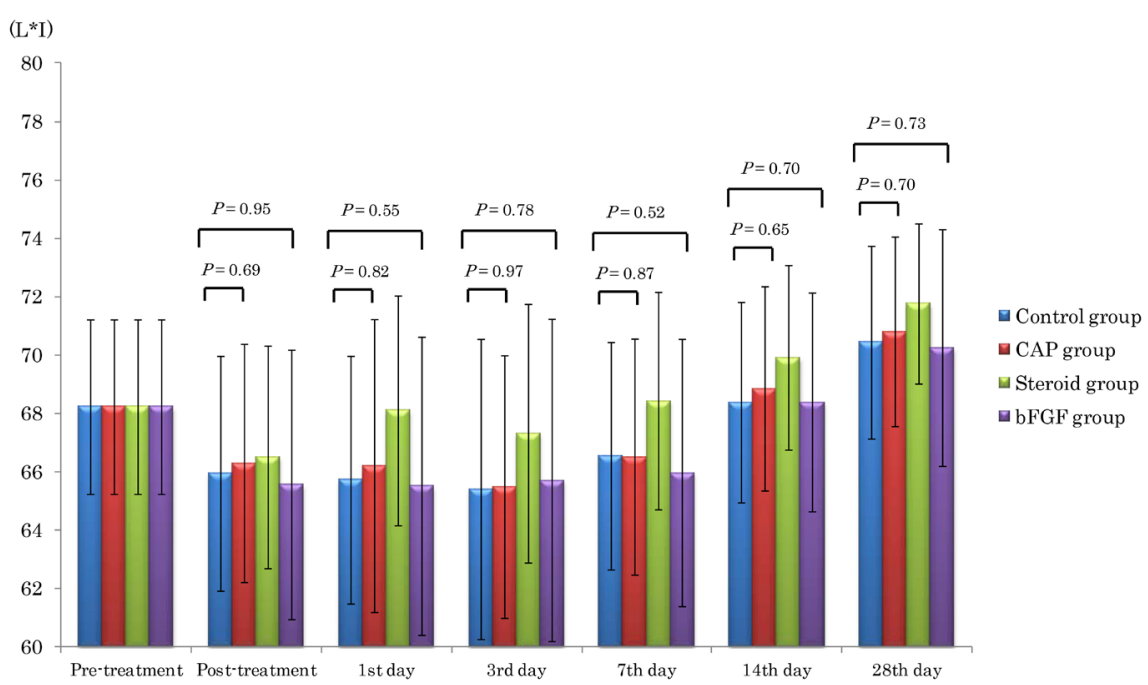

Figure 2. Time-course transition of lightness index $\left(L^{\star} I\right)$. At the end point on Day 28, skin lightness was higher than that before treatment in all groups. The steroid group had the highest skin lightness at all time points. However, no significant differences were found among the 4 groups by using one-way analysis of variance (ANOVA). Also, Mann-Whitney $U$ test showed no significant difference between the Control group and CAP group.

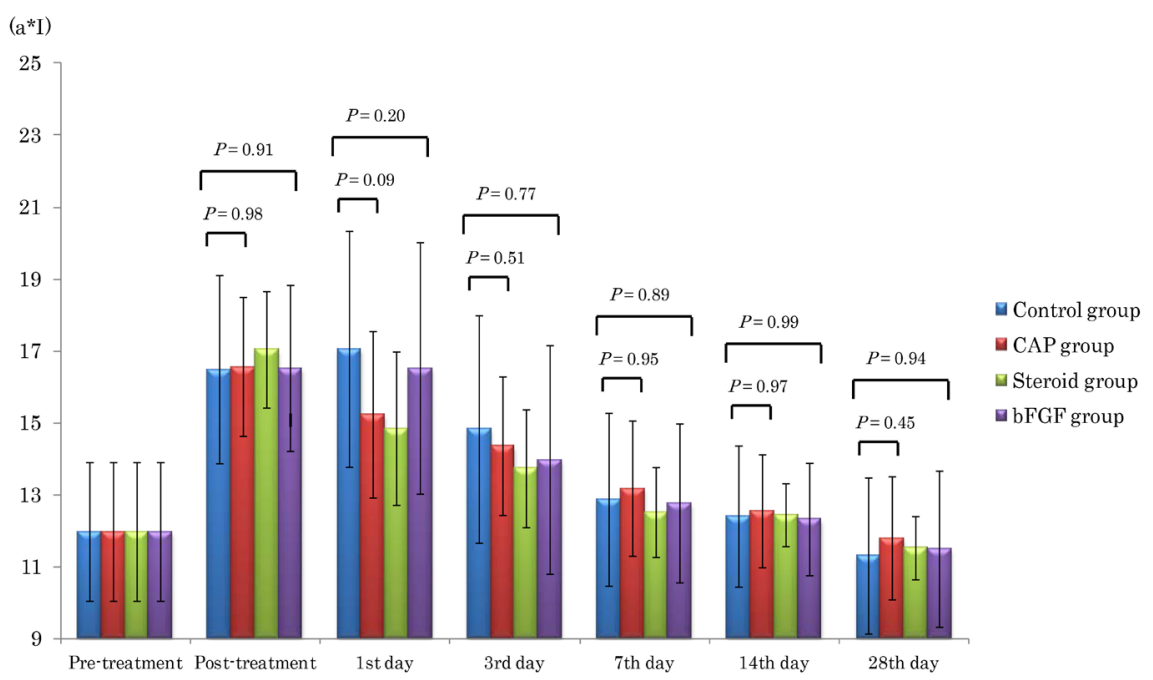

Figure 3. Time-course transition of $a^{\star} \operatorname{Index}\left(a^{\star} I\right)$. At Day 1 , the $a^{\star} I n d e x$ of the control group indicated greater redness $\left(a^{\star} I=17.0\right)$ compared with that immediately after treatment $\left(\mathrm{a}^{\star} \mathrm{I}=16.5\right)$, that of the bFGF group remained the same $\left(\mathrm{a}^{\star} \mathrm{I}=16.5\right)$, and the plasma $\left(a^{\star} I=15.2\right)$ and steroid groups $\left(a^{\star} I=14.8\right)$ had reduced redness. From Day 3 , all 4 groups exhibited similar time-course improvement. However, no significant differences were found among the 4 groups by using one-way analysis of variance (ANOVA). Also, Mann-Whitney $U$ test showed no significant difference between the Control group and CAP group.

redness $\left(\mathrm{a}^{*} \mathrm{I}\right)$ in the regions treated with CAP compared with the control regions may be the indication of anti-inflammatory effects of plasma on acute phase wounds. 


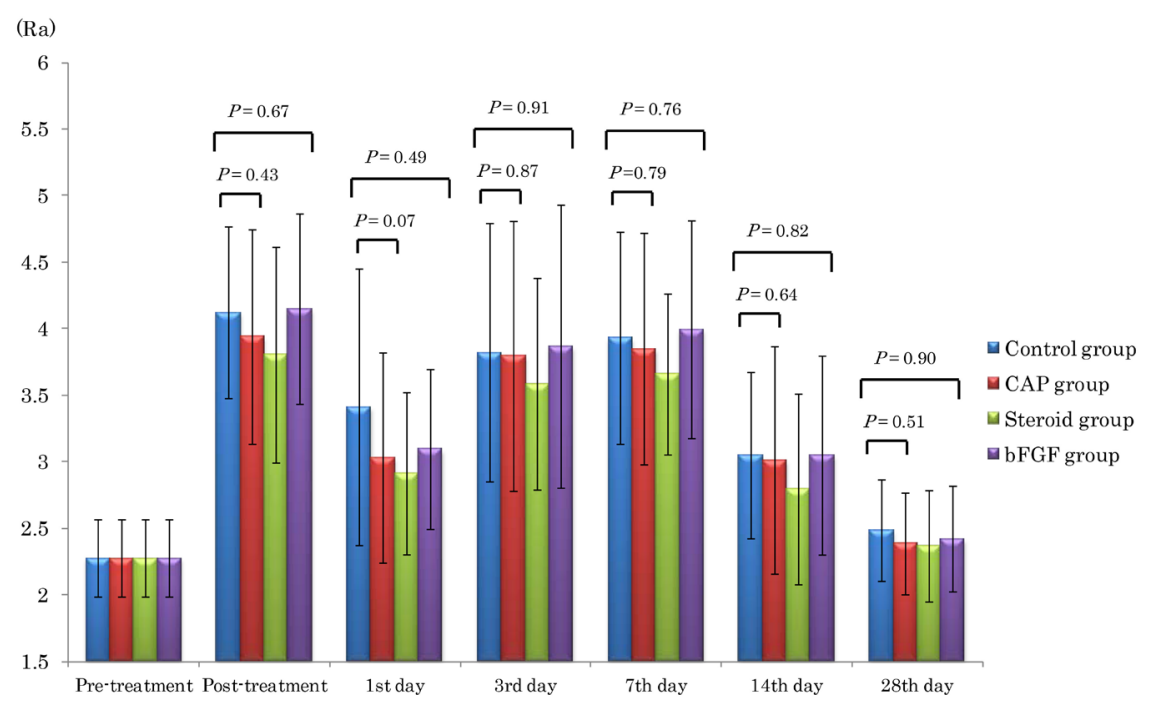

Figure 4. Time-course transition of arithmetical mean roughness ( $\mathrm{Ra})$. Ra indicated increased post-radiation swelling immediately after FXCO2 laser radiation, but improved on Day 1. The Ra increased until Day 7 along with crust formation, then decreased to a similar level as that before irradiation on Day 28. The steroid group exhibited the earliest improvement in Ra. However, no significant differences were found among the 4 groups by using one-way analysis of variance (ANOVA). Also, Mann-Whitney U test showed no significant difference between the Control group and CAP group.

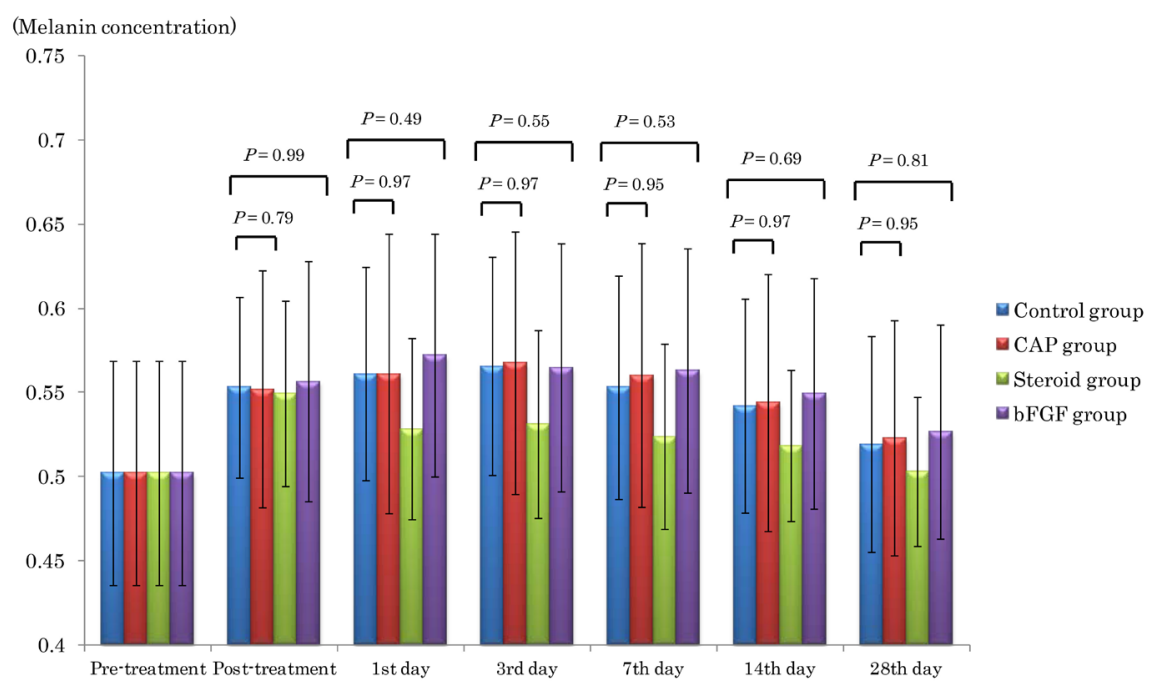

Figure 5. Time course transition of mean melanin concentration. The mean melanin concentration of the steroid group demonstrated the earliest improvement, and the other 3 groups had similar time course changes. No groups had severe hyper-pigmentation. No significant differences were found among the 4 groups by using one-way analysis of variance (ANOVA). Also, Mann-Whitney $U$ test showed no significant difference between the Control group and CAP group.

The process of wound healing is characterized by four continuous, overlapping, and precisely programmed phases: 1) hemostasis, 2) inflammation, 3) proliferation liferation, and 4) remodeling [18] [19]. During all these phases, plasma-derived activation species activate growth factors. Plasma contains many 


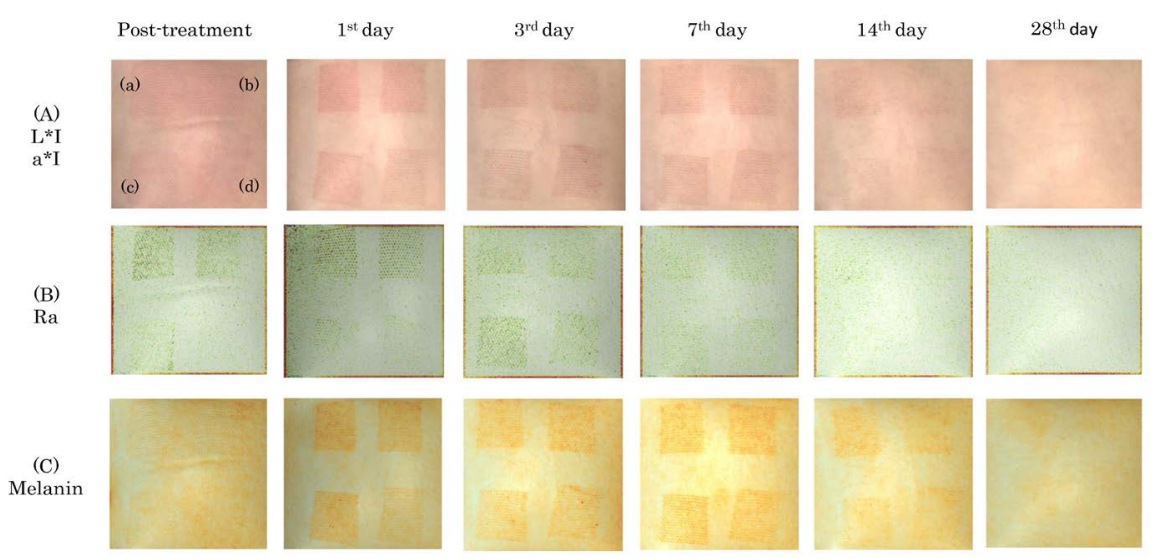

Figure 6. Representative time-course changes of the irradiated wound imaged by a $3 \mathrm{D}$ skin analyzer. (a)Control group, (b) plasma group, (c) bFGF group, (d) steroid group (A) Lightness and redness $\left(L^{\star} I\right.$ and $\left.a^{\star} I\right),(B)$ arithmetical mean roughness $(R a),(C)$ mean melanin concentration.

Table 1. Clinical findings at 7, 14, 28 days, and 1 year after irradiation using a fractional $\mathrm{CO}_{2}$ laser.

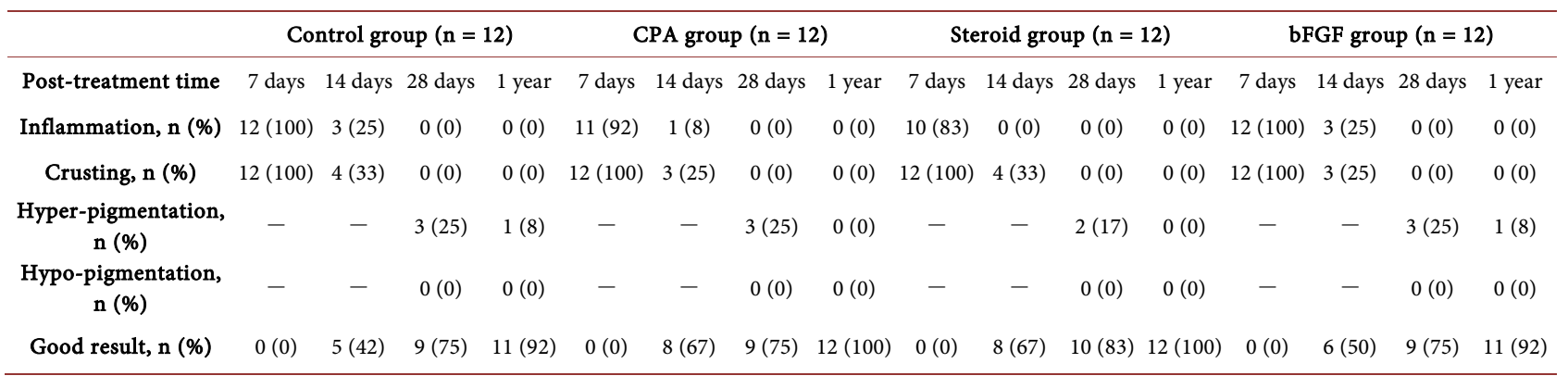

Abbreviations: CAP, cold atmospheric pressure plasma; bFGF, basic fibroblast growth factor; -: Not calculated because observation period is too short.

neutral molecules, ion species, and radical species. Nitric oxides are generated when the atmosphere is used as a source for plasma. Nitric oxide (NO) is an inorganic compound and a free radical with unpaired electrons with a half-life of 3 - 6 s. It is colorless and odorless at room temperature, and insoluble in water. In 1987, NO was found to play a major role in endothelium-derived relaxing factor, which controls blood flow, as blood flow improved after plasma irradiation [20]. Plasma can be used for redox stimulation of the skin by strongly affecting the keratinocyte transcriptome and proteome via antioxidant pathways in vitro. Both in vitro and ex vivo, plasma treatment enhances the proliferation rate of skin cells, which is highly important for wound closure. Thus, plasma alters the redox balance in skin cells, which positively affects their growth and the secretion of growth factors [1]. As medical plasma for skin treatment is simple and safe, with its efficacy confirmed, it is considered a promising treatment, for which future development is expected.

\subsection{CAP Treatment Promoting Wound Healing after Invasive Laser Irradiation}

Broad-band light treatment has been known to restore gene expression patterns 
of photoaged and intrinsically aged human skin [21]. Fractional photo-thermolysis was one of the concepts for skin restoration reported by Manstein et al. in 2004 [22], which enables effective resurfacing of the damaged skin leading to wound healing action by coagulating and degenerating the damaged skin tissues based on the non-specific caloric response of laser light. On the other hand, reactive oxygen species generated during laser irradiation on the skin involve adverse effects such as inducing erythemaor pigmentation. Fujimoto et al. applied 12 different laser therapies on 4-week-old hairless mice, and reported increased reactive oxygen species of relative superoxide anion $\left(\mathrm{O}_{2}^{-}\right)$and hydroxyl $\left(\mathrm{OH}^{-}\right)$. They demonstrated increases of both $\mathrm{O}_{2}^{-}$and $\mathrm{OH}^{-}$in the animals irradiated with a $\mathrm{CO}_{2}$ fractional laser $(10,600 \mathrm{~nm})$ similar with our experiment parameters, compared with the control group [10]. In order to inhibit oxidative skin damage after laser therapy, several post-treatment methods have been investigated. In Japan, topical application of ointments including steroids or petrolatum [9], bFGF preparation sprays, or gels containing fullerene [10] are generally applied as post-radiation treatment, as well as protection from solar radiation. In other countries, occlusive dressing material, beta-sitosterol ointment [9], MAS063DP, and $0.02 \%$ triamcinolone acetonide lotion [23] have been used in addition to the treatments applied in Japan. However, a standard treatment has yet to be established, and the choice of treatment protocols depends on each institution and the treating surgeon.

In our study, although no significant difference was observed in any evaluation item at the end point, the CAP group had accelerated anti-inflammatory activity, as the $\mathrm{a}^{\star} \mathrm{I}$ of the CAP group was less than that of the control group at Day 1. Furthermore, the CAP group had earlier improvement of swelling compared with the control group based on the Ra which indicates morphological wound healing. The time course transition of these values in the CAP group was similar with that of the steroid group, indicating the efficacy of CAP was equivalent to that of steroids. These results are considered to be due to the skin tissue activation effects of CAP.

Regarding plasma treatment for burn wounds, Hirata et al. reported that generation of active nitrogen in a gas phase, such as $\mathrm{NO}$ and nitrogen dioxide $\left(\mathrm{NO}_{2}\right)$, by the surface reaction of tissue via electrochemical stimulus activates growth factors and accelerates healing [2]. On the other hand, regarding bFGF, the $\mathrm{a}^{\star} \mathrm{I}$ and Ra of the CAP group had a similar time course change with the control group. Although plasma has been confirmed to improve the proliferation of granulation in wounds [24], data for its anti-inflammatory activity are limited.

The primary and secondary outcomes did not significantly differ among all groups in our study. This is because a relatively smooth natural healing process occurred in the control group as the fractional laser wounds only reached the superficial dermal layer and had a low possibility of infection. More marked differences in the time course outcomes may be noted in cases of contaminated wounds which require prevention and control of infection, or in cases of severe 
wounds. The reason for increased $L^{\star} I$ in all treated regions at Day 28 was considered to be due to the skin lightness increase as the nature of cosmetic skin rejuvenation after $\mathrm{FXCO}_{2}$ irradiation.

\subsection{Roles and Future Prospects of CAP in Medical Practice}

For the treatment of inflammatory lesions on the skin, topical ointments containing steroids are generally used. Our study also showed the best outcomes in the steroid group compared with the other groups, suggesting topical steroid application is optimum to suppress inflammation for acute wounds. However, although the incidence is low, steroidal drug use carries a risk of adverse reactions, including steroid acne, steroid rosacea, peristome dermatitis, excessive hair growth, steroid peliosis, skin atrophy, infection disease, contact dermatitis, and pigmentation. Caution is therefore needed to avoid severe burning or skin injury to the face.

In Japan, the use of bFGF is covered by national health insurance for the treatment of compression gangrene (bed sores) or skin ulcers, thus it is often applied in clinical practice. However, issues with bFGF include its high cost (approximately US $\$ 83.60$ for a $5-\mathrm{mL}$ bottle), the need for cold storage, the short effective time for consumption (within 2 weeks), and its lack of antibacterial effects. Steroids and bFGF preparations cannot be shared with other patients after unsealing a container for use, with a potential for waste of medical resources.

In the present study, plasma treatment exhibited several benefits: anti-inflammatory activity equivalent to that of steroid ointment, capability for continuous irradiation for each patient, and no side effects. Recently, continuous irradiation of the skin is technically possible using CAP. At a reduced cost and without the need for vacuum equipment, CAP allows for the target object to be directly processed with plasma without a vacuum disposal process. CAP therapy has therefore substantially improved clinical practice. The kINPen MED device used in this study was the first CAP jet apparatus to receive accreditation worldwide as a medical device (class IIa). Its reusable argon plasma jet can generate constant, non-thermal (room temperature) plasma at atmospheric pressure. Plasma treatment is therefore considered to be effective for post-fractional laser treatment, as well as general injuries.

\subsection{Limitations}

Our study had four limitations. First, the number of subjects was small, conducted as a single-center study, and the follow-up period was short. Second, the definition of the required number of subjects for data verification may not have been appropriate because there have been no previously published similar evaluation systems. Third, the treatments were administered to normal tissues of healthy subjects, and we did not conduct any invasive examinations such as harvesting sample tissues. Therefore, more objective evaluations, including cellular and molecular evaluations, were not available. Fourth, we did not include subjects treated with argon gas alone as a control; thus, we could not verify the 
effect of argon gas without plasmarization.

\section{Conclusion}

Although this study revealed no significant differences in treatment outcomes among 4 groups at each time points, CAP treatment by plasma jet accelerated wound healing via its anti-inflammatory activity. To further verify efficacy of CAP treatment, a multi-institutional, large-scale, randomized controlled trial including molecular analysis is necessary. Our study confirmed the effectiveness and safety of CAP, while avoiding the side-effects of conventional therapies; thus CAP may be a future treatment of choice in the field of medical plasma therapies.

\section{Financial Disclosures}

None of the authors received any financial support in association with this study.

\section{Ethical Statement}

This study was based on the declaration of Helsinki and approved by the institutional Ethics Committee of Tokyo City University (IRB number: 413), and all subjects provided written informed consent.

\section{Conflicts of Interest}

The authors certify that there is no actual or potential conflict of interest in relation to this article.

\section{References}

[1] Bekeschus, S., Schmidt, A., Weltmann, K.-D. and von Woedtke, T. (2016) The Plasma Jet kINPen-A Powerful Tool for Wound Healing. Clinical Plasma Medicine, 4, 19-28. https://doi.org/10.1016/j.cpme.2016.01.001

[2] Hirata, T., Kishimoto, T., Tsutsui, C., Kanai, T. and Mori, A. (2013) Healing Burns Using Atmospheric Pressure Plasma Irradiation. Japanese Journal of Applied Physics, 53, 010302. https://doi.org/10.7567/JJAP.53.010302

[3] Laroussi, M. (1996) Sterilization of Contaminated Matter with an Atmospheric Pressure Plasma. IEEE Transactions on Plasma Science, 24, 1188-1191. https://doi.org/10.1109/27.533129

[4] Graves, D.B. (2012) The Emerging Role of Reactive Oxygen and Nitrogen Species in Redox Biology and Some Implications for Plasma Applications to Medicine and Biology. Journal of Physics D: Applied Physics, 45, 263001. https://doi.org/10.1088/0022-3727/45/26/263001

[5] Ulrich, C., Kluschke, F., Patzelt, A., Vandersee, S., Czaika, V.A., Richter, H., Bob, A., Hutten, J., Painsi, C., Huge, R., Kramer, A., Assadian, O., Lademann, J. and Lange-Asschenfeldt, B. (2015) Clinical Use of Cold Atmospheric Pressure Argon Plasma in Chronic Leg Ulcers: A Pilot Study. Journal of Wound Care, 24, 196, 198-200, 202-193.

[6] Saluja, R., Khoury, J., Detwiler, S.P. and Goldman, M.P. (2009) Histologic and Clinical Response to Varying Density Settings with a Fractionally Scanned Carbon 
Dioxide Laser. Journal of Drugs in Dermatology: JDD, 8, 17-20.

[7] Manuskiatti, W., Triwongwaranat, D., Varothai, S., Eimpunth, S. and Wanitphakdeedecha, R. (2010) Efficacy and Safety of a Carbon-Dioxide Ablative Fractional Resurfacing Device for Treatment of Atrophic Acne Scars in Asians. Journal of the American Academy of Dermatology, 63, 274-283.

https://doi.org/10.1016/j.jaad.2009.08.051

[8] Chapas, A.M., Brightman, L., Sukal, S., Hale, E., Daniel, D., Bernstein, L.J. and Geronemus, R.G. (2008) Successful Treatment of Acneiform Scarring with $\mathrm{CO}_{2}$ Ablative Fractional Resurfacing. Lasers in Surgery and Medicine, 40, 381-386.

https://doi.org/10.1002/1sm.20659

[9] Lee, C.J., Whang, J.H., Lazova, R., Ciesielski, T.E., Thomson, J.G., McCarthy, T. and Persing, J.A. (2007) Growth Factor Expression with Different Wound Treatments after Laser Resurfacing. Aesthetic Surgery Journal, 27, 55-64.

https://doi.org/10.1016/j.asj.2006.12.002

[10] Fujimoto, T., Ito, S., Ito, M., Kanazawa, H. and Yamaguchi, S. (2012) Induction of Different Reactive Oxygen Species in the Skin during Various Laser Therapies and Their Inhibition by Fullerene. Lasers in Surgery and Medicine, 44, 685-694. https://doi.org/10.1002/lsm.22065

[11] Metelmann, H.-R., Vu, T.T., Do, H.T., Le, T.N.B., Hoang, T.H.A., Phi, T.T.T., Luong, T.M.L., Nguyen, T.T.H., Nguyen, T.H.M. and Nguyen, T.L. (2013) Scar Formation of Laser Skin Lesions after Cold Atmospheric Pressure Plasma (CAP) Treatment: A Clinical Long Term Observation. Clinical Plasma Medicine, 1, 30-35. https://doi.org/10.1016/j.cpme.2012.12.001

[12] Vandersee, S., Richter, H., Lademann, J., Beyer, M., Kramer, A., Knorr, F. and Lange-Asschenfeldt, B. (2014) Laser Scanning Microscopy as a Means to Assess the Augmentation of Tissue Repair by Exposition of Wounds to Tissue Tolerable Plasma. Laser Physics Letters, 11, 115701. https://doi.org/10.1088/1612-2011/11/11/115701

[13] Matias, A.R., Ferreira, M., Costa, P. and Neto, P. (2015) Skin Colour, Skin Redness and Melanin Biometric Measurements: Comparison Study between Antera ${ }^{\circledR}$ D, Mexameter ${ }^{\circledast}$ and Colorimeter ${ }^{\oplus}$. Skin Research and Technology, 21, 346-362. https://doi.org/10.1111/srt.12199

[14] Messaraa, C., Metois, A., Walsh, M., Hurley, S., Doyle, L., Mansfield, A., O’Connor, C. and Mavon, A. (2018) Wrinkle and Roughness Measurement by the Antera 3D and Its Application for Evaluation of Cosmetic Products. Skin Research and Technology, 24, No. 3. https://doi.org/10.1111/srt.12436

[15] Zhang, N., Shi, K., Hong, L., Zhao, J. and Yu, J. (2018) Antera 3D Camera: A Novel Method for Evaluating the Therapeutic Efficacy of Fractional $\mathrm{CO}_{2}$ Laser for Surgical Incision Scars. Journal of Cosmetic Dermatology, 17, No. 6.

https://doi.org/10.1111/jocd.12738

[16] Funk, W., Podmelle, F., Guiol, C. and Metelmann, H.R. (2012) Aesthetic Satisfaction Scoring-Introducing an Aesthetic Numeric Analogue Scale (ANA-Scale). Journal of Cranio-Maxillo-Facial Surgery. Official Publication of the European Association for Cranio-Maxillo-Facial Surgery, 40, 439-442. https://doi.org/10.1016/j.jcms.2011.07.018

[17] Daeschlein, G., Scholz, S., Ahmed, R., von Woedtke, T., Haase, H., Niggemeier, M., Kindel, E., Brandenburg, R., Weltmann, K.D. and Juenger, M. (2012) Skin Decontamination by Low-Temperature Atmospheric Pressure Plasma Jet and Dielectric Barrier Discharge Plasma. The Journal of Hospital Infection, 81, 177-183. 
https://doi.org/10.1016/j.jhin.2012.02.012

[18] Guo, S. and Dipietro, L.A. (2010) Factors Affecting Wound Healing. Journal of Dental Research, 89, 219-229. https://doi.org/10.1177/0022034509359125

[19] Gurtner, G.C., Werner, S., Barrandon, Y. and Longaker, M.T. (2008) Wound Repair and Regeneration. Nature, 453, 314-321. https://doi.org/10.1038/nature07039

[20] Murad, F. (2004) Discovery of Some of the Biological Effects of Nitric Oxide and Its Role in Cell Signaling. Bioscience Reports, 24, 452-474.

https://doi.org/10.1007/s10540-005-2741-8

[21] Chang, A.L., Bitter, P.H., Qu, K., Lin, M., Rapicavoli, N.A. and Chang, H.Y. (2013) Rejuvenation of Gene Expression Pattern of Aged Human Skin by Broadband Light Treatment: A Pilot Study. The Journal of Investigative Dermatology, 133, 394-402. https://doi.org/10.1038/jid.2012.287

[22] Manstein, D., Herron, G.S., Sink, R.K., Tanner, H. and Anderson, R.R. (2004) Fractional Photothermolysis: A New Concept for Cutaneous Remodeling Using Microscopic Patterns of Thermal Injury. Lasers in Surgery and Medicine, 34, 426-438. https://doi.org/10.1002/lsm.20048

[23] Lueangarun, S. and Tempark, T. (2018) Efficacy of MAS063DP Lotion vs. $0.02 \%$ Triamcinolone Acetonide Lotion in Improving Post-Ablative Fractional $\mathrm{CO}_{2}$ Laser Resurfacing Wound Healing: A Split-Face, Triple-Blinded, Randomized, Controlled Trial. International Journal of Dermatology, 57, 480-487. https://doi.org/10.1111/ijd.13922

[24] Akita, S., Akino, K., Imaizumi, T., Tanaka, K., Anraku, K., Yano, H. and Hirano, A. (2006) The Quality of Pediatric Burn Scars Is Improved by Early Administration of Basic Fibroblast Growth factor. Journal of Burn Care \& Research: Official Publication of the American Burn Association, 27, 333-338.

https://doi.org/10.1097/01.BCR.0000216742.23127.7A 